JOURNAL OF

SYNCHROTRON

RADIATION

ISSN 1600-5775

Received 5 August 2020

Accepted 8 December 2020

Edited by S. M. Heald, Argonne National Laboratory, USA

Keywords: XAS; XANES; electrochemistry; spectroelectrochemistry; photoreduction; copper complexes; additive engineering.

Supporting information: this article has supporting information at journals.iucr.org/s

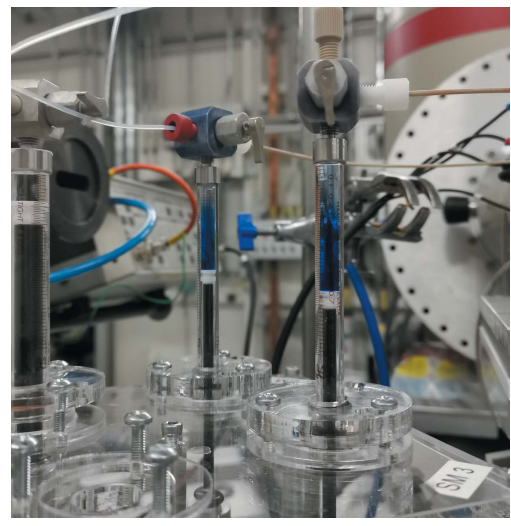

C 2021 International Union of Crystallography

\section{Redox state and photoreduction control using X-ray spectroelectrochemical techniques - advances in design and fabrication through additive engineering}

\author{
Stephen Peter Best, ${ }^{\mathrm{a} *}$ Victor A. Streltsov, ${ }^{\mathrm{b}}$ Christopher Thomas Chantler, ${ }^{\mathrm{c}}$ \\ Wangzhe Li, ${ }^{d}$ Philip A. Ash, ${ }^{e}$ Shusaku Hayama ${ }^{f}$ and Sofia Diaz-Moreno ${ }^{f}$

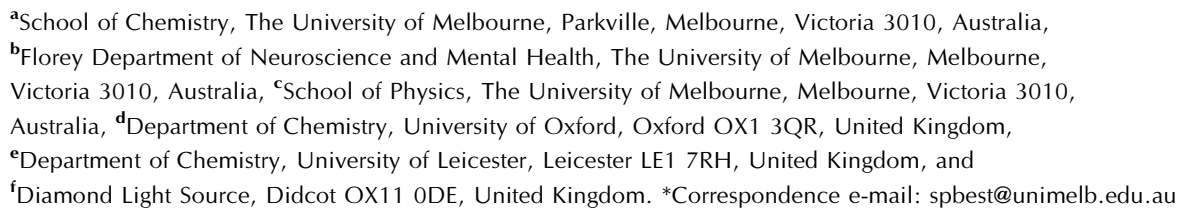

The design and performance of an electrochemical cell and solution flow system optimized for the collection of X-ray absorption spectra from solutions of species sensitive to photodamage is described. A combination of 3D CAD and 3D printing techniques facilitates highly optimized design with low unit cost and short production time. Precise control of the solution flow is critical to both minimizing the volume of solution needed and minimizing the photodamage that occurs during data acquisition. The details of an integrated four-syringe stepper-motor-driven pump and associated software are described. It is shown that combined electrochemical and flow control can allow repeated measurement of a defined volume of solution, $100 \mu \mathrm{l}$, of samples sensitive to photoreduction without significant change to the X-ray absorption near-edge structure and is demonstrated by measurements of copper(II) complexes. The flow in situ electrochemical cell allows the collection of high-quality X-ray spectral measurements both in the near-edge region and over an extended energy region as is needed for structural analysis from solution samples. This approach provides control over photodamage at a level at least comparable with that achieved using cryogenic techniques and at the same time eliminates problems associated with interference due to Bragg peaks.

\section{Introduction}

Structure elucidation of non-crystalline samples using X-ray absorption spectroscopy (XAS), from analysis of the X-ray near-edge structure (XANES) or extended X-ray absorption fine-structure (EXAFS) regions of the spectrum, plays a critical part in the understanding of materials where the property of interest is associated with an element which exclusively occurs at a specific site (Frenkel \& van Bokhoven, 2014; Bunker, 2010; Parsons et al., 2002). If the element has a moderate atomic number, then the X-ray energy is highly penetrating and highly specific results can be obtained from dilute samples. Metalloproteins present a matrix comprised mainly of low atomic number elements where protein function is often associated with a transition metal. If present in a single site, the coordination of the metal can be determined with greater precision using EXAFS than for many protein X-ray structures (Zhang, 2019; Tamura et al., 2013; Cotelesage et al., 2012; Shima et al., 2008; Grunwaldt \& Clausen, 2002; Zhang et al., 1999) and this advantage can be extended to small-molecule structure determination when the analysis incorporates data corrected for systematic errors with statistically valid 
error propagation (Trevorah et al., 2020; Schalken \& Chantler, 2018; Bourke et al., 2016).

Since there is not a requirement for crystalline samples, EXAFS permits structure determination of solvated species under conditions relevant to their function. This is particularly important for polypeptides where protein folding or acid/base reactions of the sidechains can impact on the active site structure. Despite the opportunity to make measurements from samples in the solution state, practical considerations have largely contributed to the predominance of XAS measurements from metalloproteins and related samples to be conducted at temperatures well below that of liquid nitrogen. For measurements from first-row transition metals at the $K$ edge, photoreduction can destroy the integrity of the sample on a timescale shorter than that needed for the measurement of an absorption spectrum. The impact of unwanted photoredox chemistry is particularly pronounced for solution samples at ambient temperatures, owing to diffusion of the primary and secondary products of photoionization. These problems may be addressed by employing solution jets, where reports of $L$-edge spectra (Kubin et al., 2018) and X-ray freeelectron laser (Chatterjee et al., 2019) measurements give a measure of the current status. Even with highly optimized drop-on-demand approaches sample volumes of $c a 5 \mathrm{ml}$ per spectrum are needed (Chatterjee et al., 2019). Rapid freezing of the solution, after the addition of cryoprotectants designed to reduce crystallization, significantly reduces the sample volume requirements and can produce a sample which is more robust in terms of photoreduction, although problems commonly persist for absorbers such as $\mathrm{Cu}$, As and Se (George et al., 2012). This is well illustrated by recent studies into the impact of photoreduction of $\mathrm{Cu}$-bound amyloid $\beta$ peptide $(\mathrm{CuA} \beta)$ samples which have examined both the time/ flux dependence of sample degradation and the impact of cryoprotectants (Summers et al., 2019; Stellato et al., 2019; Streltsov et al., 2008).

Against this backdrop we have sought to develop methods which allow the measurement of XAS spectra at hard X-ray energies $(>4 \mathrm{keV})$ from small volumes $(<1 \mathrm{ml})$ of solution samples at ambient temperature under potentiostatic control. Early reports of the sulfur $K$-edge and molybdenum $L$-edge EXAFS of static $N$-methyl formamide solutions of the ironmolybdenum cofactor of nitrogenase $(340 \mu \mathrm{l})$ under electrochemical control demonstrate the feasibility of the approach, albeit for samples which are stable over the long electrosynthesis timescale $(10 \mathrm{~min})$ and are not sensitive to photodamage (Schultz et al., 1993). More recently we have described a small-volume flow electrochemical cell which is suitable for biological samples [e.g. cytochrome $c$ (Best et al., 2016)] and for radiation-sensitive $\mathrm{CuA} \beta$ complexes (Streltsov et al., 2018). A limitation to the more widespread use of the approach is the requirement of significant workshop facilities for the implementation of that design. Advances in additive manufacturing (3D printing) when coupled with computer assisted design (CAD) packages provide a path to more highly optimized cell designs which can be fabricated at low cost. In this contribution we describe the CAD-based design and 3D printed XAS spectroelectrochemical (XAS-SEC) cells and the design and software control of an integrated stepper motor syringe pump. We also explore the strategies that these methods bring to the control of the redox state of the sample and demonstrate the data quality obtained using XAS-SEC methods.

\section{Experimental}

X-ray absorption spectra were measured using the I20Scanning wiggler beamline of Diamond Light Source (DLS) (Diaz-Moreno et al., 2018). The four-bounce monochromator (Hayama et al., 2018) equipped with $\mathrm{Si}(111)$ crystals gives an energy resolution, $\Delta E / E$, better than $1.4 \times 10^{-4}$ with a flux $>10^{12}$ photons s ${ }^{-1}$ and a focal spot size at the sample position of $0.4 \mathrm{~mm}(\mathrm{H}) \times 0.3 \mathrm{~mm}(\mathrm{~V})$ FWHM. A Canberra 64-pixel Monolithic Segmented Hyper Pure Germanium Detector (HPGe) partnered with the Xspress4 digital pulse processor (Dennis et al., 2019) was used to measure the fluorescence signal emitted from the solution.

Solutions of $\left[\mathrm{CuCl}(\mathrm{bpy})_{2}\right] \mathrm{Cl}$-hydrate were prepared with ca $2 \mathrm{~m} M$ copper concentration in saline phosphate buffer (PBS, $0.137 M \mathrm{NaCl}, 0.01 M \mathrm{Na}_{2} \mathrm{HPO}_{4}, 0.002 M \mathrm{KH}_{2} \mathrm{PO}_{4}$, $0.003 \mathrm{M} \mathrm{KCl}$ ). Solutions were degassed in an MBraun glove box and transferred to gas-tight syringes and valves (Hamilton). The three syringes, one containing supporting electrolyte, PBS, for the counter electrode (CE) and a pair of syringes for the working electrode (WE) were removed from the glove box and connected to the XAS-SEC cell. The narrow-bore 1/16-inch Teflon tubing was flushed with dinitrogen before connecting to the filled syringes. With appropriate fittings it is possible to establish a closed volume of cell and syringes without measurable dioxygen contamination.

Electrochemical measurements were conducted using a PalmSens 4 compact electrochemical interface. The $10 \mathrm{~V}$ compliance voltage and $30 \mathrm{~mA}$ maximum current were compatible with the demands of the large-surface-area WE of the XAS-SEC cell. The cell incorporated a model ET072 (eDAQ) $2 \mathrm{~mm}$-diameter leakless $\mathrm{Ag} / \mathrm{AgCl}$ miniature reference electrode (RE) and the porous WE and CE were fashioned from 500 pores per inch reticulated vitreous carbon (RVC; Destech Corporation), which had been made hydrophilic by immersion in concentrated sulfuric acid then thoroughly rinsed with MilliQ water and buffer. The space between the electrodes was filled with glass beads (45-60 mesh, Selby).

The design criteria for the XAS-SEC cell are described in the following section and were implemented using the opensource software OpenSCAD (OpenSCAD, 2020). This supports the output of the STL files needed for 3D printing. A Connex3 Objet350 printer with UV-cured ink (Vero, exo-1,7,7trimethylbicyclo[2.2.1]hept-2-yl acrylate, camphene) and a water-soluble support (acrylic acid, 2-hydroxyethyl ester) produced objects with high geometric precision suitable for working with aqueous solutions. The UV-cured polymer was found to contain low concentrations of copper and a detectable $\mathrm{Cu}$ edge could be observed for direct irradiation of that 
material. For the cell body this does not present a significant problem since the X-ray flux after passage through the sample is attenuated to the order of $1 \%$ and any fluorescence from that source directed towards the detector will be further attenuated by the sample. The mounting plate will be exposed to scattered X-rays; therefore, the fluorescence has an unattenuated path to the detector elements and presents the most serious risk of contamination of the spectra for lowconcentration samples. The thermoset printing material ULTEM does not contain measurable concentrations of copper and, though unsuitable for printing the main body of the XAS-SEC cell, could be used to print the front mounting plate satisfactorily [Fig. 1(a)]. Measurements from the supporting electrolyte in an XAS-SEC cell comprising UVcured polymer printed cell body and CE holder and ULTEM printed mounting plate did not give an observable copper signal. Owing to the complexity of the objects it was not
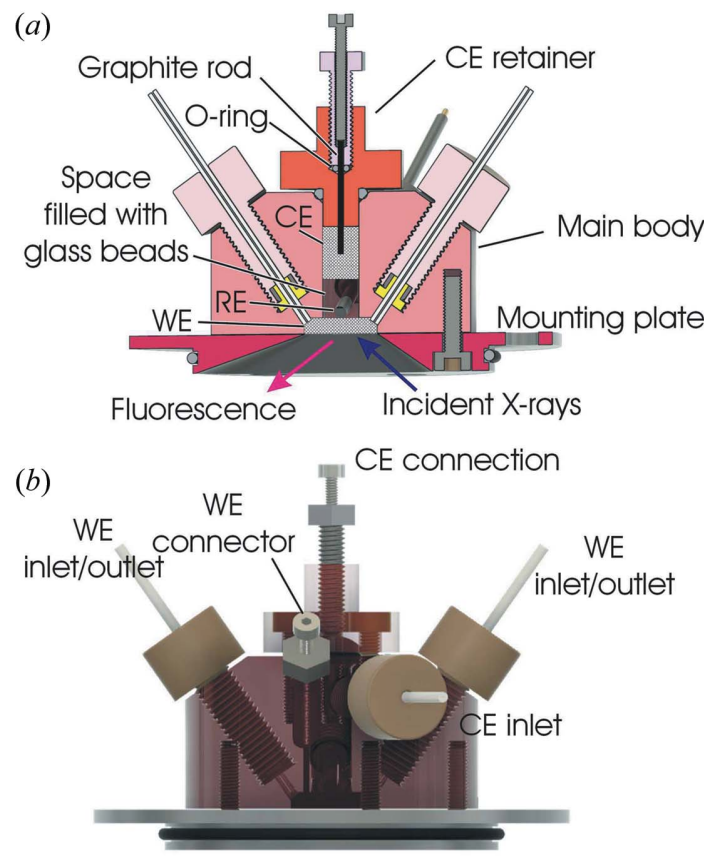

(c)

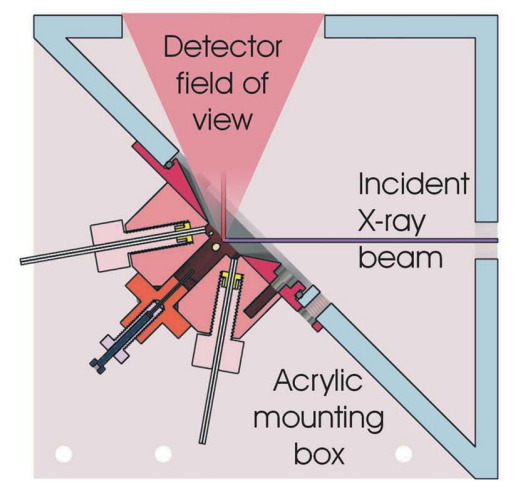

Figure 1

(a) Cross-sectional view of the XAS-SEC cell showing entry and exit ports, the positioning of the working (WE), counter (CE) and reference (RE) electrodes and the electrical connection to the CE. (b) 3D-CAD view of the assembled cell. (c) Cross-sectional view of the XAS-SEC cell mounted onto an acrylic box which is sealed with Kapton windows and can be flushed with $\mathrm{He}$ and thereby minimize air scatter. practicable to program explicitly the threads when using OpenSCAD so they were manually tapped into the printed objects. More highly featured CAD programs allow direct printing of threaded components. The technical figures for this communication were generated using the program Autodesk Fusion 360.

The physical characteristics and durability of the construction material impact on the reliability and suitability of the cell for different applications. The UV-cured polymer is rigid, but brittle, and has poor non-aqueous solvent compatibility. The internal threaded sections are vulnerable to stripping and manually tapped threads could be used on three to four occasions. The durability of the threaded components may be improved by additional UV-curing of the cells or by explicit printing of the threaded sections. Thread inserts present an alternative strategy commonly used when tapped sections are needed in soft materials. That strategy would require more substantial redesign to accommodate the larger-diameter holes needed for the inserts. However, it is important to note that the limitation in repeat use of the current cell is offset by the comparatively low unit cost of the 3D-printed cell.

The four-axis stepper motor syringe pump was constructed using 0.9 JK57HM41-1006 (Changzhou JKongmotor Co., Ltd) stepper motors coupled to a 4 turn $\mathrm{mm}^{-1}$ lead screw (Thorlabs F6MSS100-sp). A matching threaded bushing (Thorlabs F6MSSN1P) was attached to a $20 \mathrm{~mm} \times 20 \mathrm{~mm} \times$ $100 \mathrm{~mm}$ acrylic block. The elimination or minimization of backlash in the flow system is a key design property as this defect can cause pressure changes in the WE and lead to a period whereby the solution is static when the flow direction is reversed. Backlash was minimized using guide rods $(8 \mathrm{~mm}$ diameter silver steel ground shaft rod) which were offset by $40 \mathrm{~mm}$ from the beam axis [Fig. 2(a)]. The mounting plates for the stepper motors, alignment guides and syringes were laser cut from an $8 \mathrm{~mm}$-thick acrylic sheet. Further details of the construction of the syringe pump assembly are given in Section S1 of the supporting information.

A KTA-290 USB Serial Stepper Motor Controller and SFC027 Big Easy Driver boards were used to control the stepper motors. Purpose-built software, written using Microsoft Visual Studio, allowed control of the four independent syringe pumps. This supported the operation of the XAS-SEC cell in linear-flow and pulsed-flow modes with automatic reversal of the direction of net solution flow after reaching programmable software limits (Section S2 of the supporting information). At the highest resolution (16 microsteps per step) the linear resolution was 25.8 microsteps $\mu \mathrm{m}^{-1}$ and backlash $<2 \mu \mathrm{m}$.

\section{Results and discussion}

\subsection{XAS-SEC cell}

Whilst the basic principles of the design of the XAS-SEC cell have been described previously (Best et al., 2016), the coupling of CAD with 3D printing can allow the realization of more intricate designs and inexpensive unit costs. Although there are a range of commercial CAD programs, open source 
(a)
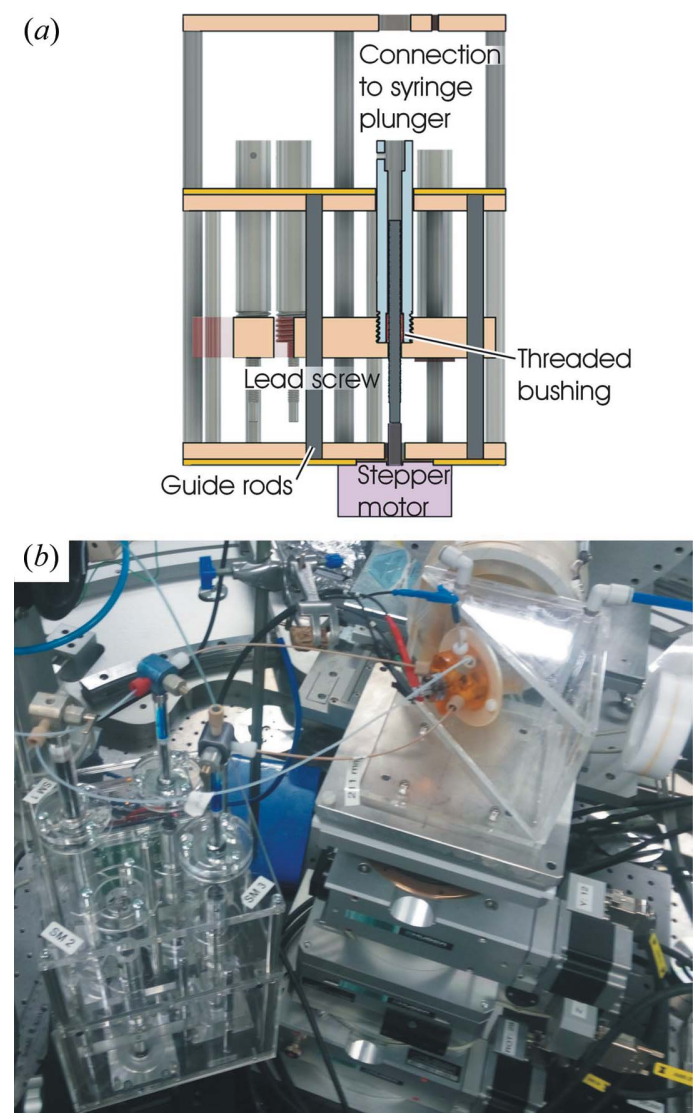

Figure 2

(a) Cross-sectional schematic of a stepper motor syringe driver assembly. (b) Syringe pump assembly combined with the XAS-SEC mounted on the beamline sample stages.

software such as OpenSCAD can provide sufficient functionality to allow the design of component parts and generation of files for printing and 3D visualization. The STL files of the main body, CE holder and mounting plate are available in Section S3 of the supporting information.

Critical requirements for the cell design are the minimization of the solution volume needed for operation and optimization of the reliability of the cell assembly in terms of establishing electrical connections and elimination of leaks. The electrochemical cell consists of porous $\mathrm{WE}$ and $\mathrm{CE}$ fashioned from $\mathrm{RVC}$ with a $2 \mathrm{~mm}$-diameter $\mathrm{Ag} / \mathrm{AgCl} \mathrm{RE}$ positioned with its junction close to the WE. Mixing of the solution in the WE and CE is minimized by packing the space between the electrodes with glass beads with particle sizes larger than the galleries in the $\operatorname{RVC}(>100 \mu \mathrm{m})$. While materials such as Nafion can provide ion transport while blocking diffusion of large and/or anionic species, the restrictions Nafion provides to mass transport complicate filling and flushing the current design of the flow cell. Our previous investigations (Streltsov et al., 2018; Best et al., 2016) suggest that, after flushing the WE and flow system, solute diffusion into the glass bead layer in contact with the WE is sufficiently low for there to be no significant variation in the solute concentration during flow experiments. The WE is enclosed by a Teflon-coated Kapton film sandwiched between the main body and the mounting plate. Leakage between the Kapton film and the cell body could be eliminated by the use of a Teflon gasket, fashioned by cutting a rectangle from Teflon tape with dimensions matching the WE slot. Electrical connections to the RVC electrodes are achieved using a graphite rod ( $0.9 \mathrm{~mm} \mathrm{HB}$ pencil lead) which is O-ring-sealed into the main body (WE) or CE retainer [Fig. 1(a)]. A Nylon M4 screw with a central M2 tap is used to compress the O-ring, then an M2 screw is used to push the graphite rod into the RVC and provide electrical contact. The 1/16-inch tubing which facilitated solution flow through the electrodes was sealed into the XAS-SEC cell using flangeless liquid chromatography fittings (Upchurch Scientific).

During operation the cell is connected to the three syringes, tested for leaks and degassed by a flow of dinitrogen. Deoxygenated supporting electrolyte is flowed into the CE entry port and out of the WE inlet/outlets and the electrochemical performance of the cell is tested. The supporting electrolyte in the circuit between the two WE syringes is replaced by flowing the solute solution to waste. It was found that $50 \mu \mathrm{l}$ in addition to the dead volume of the taps, tubing and WE volume was sufficient to provide an enclosed volume that is not affected by significant concentration gradients.

\subsection{The flow system}

Successful operation of the cell requires fine control over both the volume and rate of solution flow and, though commercial syringe pumps can serve this purpose, there can be complications associated with programming the flow pattern, acceleration profile and synchronization of independent syringes. By using a modular approach, it is comparatively straightforward to build the flow system with optimized mechanical properties and integrate the control software. The stepper motor (SM) assembly is shown in Fig. 2. Successful implementation of pulsed-flow modes of operation (vida infra) require smooth acceleration/deceleration and minimal backlash associated with a change in flow direction.

Mechanical coupling of the gas-tight syringes to the SM assembly [Fig. 2(b)] is relatively straightforward and has been optimized to facilitate coupling the filled syringes (Section S1 of the supporting information). In order to minimize dioxygen concentrations, the syringes are filled in a glove box and the solution isolated until the tubing and the XAS-SEC cell were purged with dinitrogen gas.

A commercial four-axis stepper motor controller with builtin acceleration/deceleration and USB interface (KTA-290) provided a convenient means of controlling the stepper motors. Since the concentration of solute needed for the collection of extended XAS spectra with a good signal-tonoise ratio in a reasonable timeframe $(<1-2 \mathrm{~h})$ is in the millimolar regime, it is often challenging to obtain $1 \mathrm{ml}$ of many protein samples. Consequently, solution management must be both precise and reliable. Software has been written to allow (1) the setting of software limits for each of the syringes, (2) linear flow of designated solution volumes for both open (flow to waste) and closed (syringe-to-syringe 
transfer) configurations, (3) pulsed-flow operation of a matched pair of syringes with control over both the forward and the return flow volume for each pulse, and (4) reversal of the pulsed-flow direction on reaching the syringe limits or the net flow if a volume of solution is specified. Further details of the software are provided in Section S2 of the supporting information.

The flow properties of the sample are impacted by solution viscosity and the characteristics of the tubing used in the experiment; moreover, for large biomolecules it is necessary to ensure that the solution flow does not generate shear forces sufficient to denature the protein. The objectives of minimizing the solution dead volume and the pressures within the system together contribute to the selection of the tubing. For aqueous solutions, 0.01-inch or 0.02-inch Teflon/PEEK/ PEEKsil tubing has been used successfully. The introduction of air bubbles during pulsed-flow operation can be a problem and is caused by failure of the seals of the tubing or the gastight syringe and will be exacerbated by the negative pressures generated during pulsed flow. In addition to attention to the plumbing, it is necessary to ensure well matched operation of syringe pumps and that the programmed flow, including the acceleration/deceleration profile, can be supported by the system.

\subsection{XAS spectroscopy}

In order to minimize air scatter and to fix, reliably, the angle between incident beam and the front surface of the WE at $45^{\circ}$, the XAS-SEC cell was mounted onto a He-flushed acrylic box [Fig. 1(c)]. With this orientation the WE can be translated over a $5 \mathrm{~mm} \times 1.2 \mathrm{~mm}(\mathrm{H} \times \mathrm{V})$ area while maintaining an unattenuated path of the fluorescent photons to the detector [Fig. 1(a)]. This is sufficient for a $4 \times 2$ grid of non-overlapping sampling positions of the $0.4 \mathrm{~mm} \times 0.3 \mathrm{~mm}(\mathrm{H} \times \mathrm{V})$ beam of I20-Scanning, which may be used to assess beam-induced damage to the electrode.

Critical to the experiment design is the identification of a combination of flow and X-ray flux parameters which allow the measurement of spectra while minimizing photodamage. In order to minimize the quantity of sample dedicated to this task a pulsed sample mode of operation was used and the direction of solution flow reversed after passage of a net flow of 50-100 $\mu \mathrm{l}$. In this way, several combinations of net flow per pulse, residence time and X-ray beam intensity can be tested. Once a suitable set of parameters is identified, and the constancy of the spectra with a lower beam flux confirmed, the cell is moved to illuminate a fresh area of the electrode and the 'setup' solution flowed beyond the sampling point of the cell.

For the flowing sample, photodamage to the sample does not appear to depend simply on the photon dose. If the concentration of photoredox products is maintained at a low level the photochemical reactions can be reversed by chemical or electrochemical reactions. Similar spectra could be obtained from $100 \mu \mathrm{l}$ of solution passed through the sample point ten times and from the linear passage of $1 \mathrm{ml}$ solution with the same pulse characteristics (i.e. both with and without
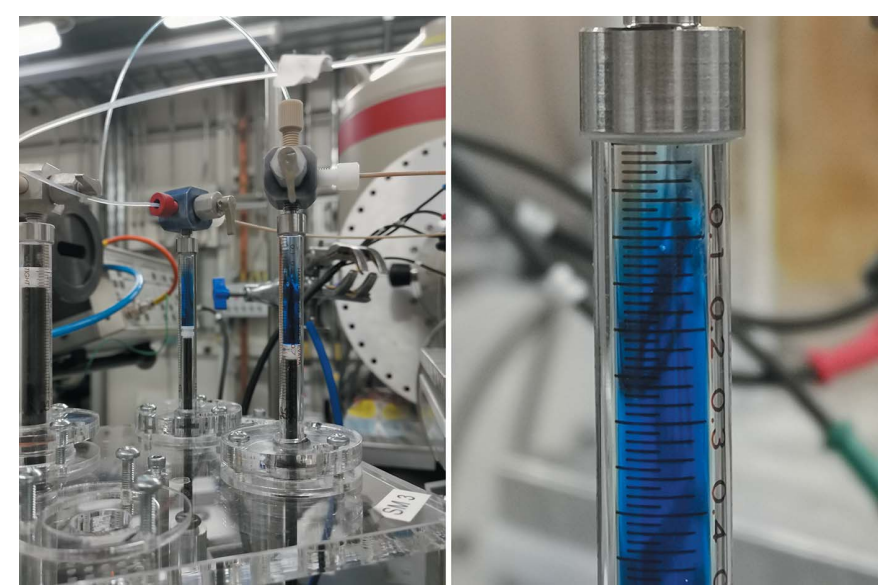

Figure 3

Solutions of a copper(II) complex together with the redox mediator DCIP (dichlorophenolindophenol) following multiple passes of $100 \mu \mathrm{l}$ of the XAS-SEC cell at reducing potentials. The oxidized form of DCIP is an intense blue and the reduced form is colourless. A close-up view of the receiver syringe is also shown.

reversing the direction of net flow). There is some mixing of the solution in the syringe during the pulsing of the solution, as may be seen in cases where the redox reaction leads to a change in colour of the solution (Fig. 3). The reproducibility of the spectra can be tested by (1) recovery of the starting spectrum by application of an appropriate potential and (2) repeat measurements from a 'fresh' part of the solution contained within the syringe.

The quality of XAS data depends on both the compositional stability of the sample and the stability of the cell relative to the beam. In particular, changes to the geometry of the front window of the WE due to varying pressure during pulsed-flow operation introduce higher-frequency noise into the spectra and longer timeframe distortions would be associated with any changes in the composition of the sample over the course of the measurement. We previously established that with properly matched syringes there is no degradation of the signal-tonoise ratio for pulsed-flow compared with static or linear-flow solutions (Streltsov et al., 2018; Best et al., 2016). The stability of the sample and the state of the electrode surface must be assessed in the context of the total measurement time, the photon flux and flow characteristics of the solution.

For the solute species, a good starting position is to set a pulse with forward and reverse flows of $0.5 \mu \mathrm{l}$ and $0.4 \mu \mathrm{l}$ and a frequency of $2 \mathrm{~Hz}$. This would give a net flow of the solution front of $1 \mathrm{~mm}$ in $50 \mathrm{~s}$. Since the I20 scanning beam height is $20 \%$ of that of the WE, mixing of the solution during pulsing will give the solution an average exposure time of $c a 5 \mathrm{~s}$ for a $1 \mathrm{~mm}$ beam width. These flow parameters give a net flow of $100 \mu \mathrm{l}$ of solution in $16.7 \mathrm{~min}$. Starting with a more attenuated beam, XANES spectra were monitored as a function of the photon flux and systematic differences in the spectra were used to set an appropriate beam flux. An example of the variation of the spectra in response to a change in beam attenuation is given for PBS solutions of $\left[\mathrm{CuCl}(\text { bipy })_{2}\right] \mathrm{Cl}$. hydrate. The crystal structure indicates that the complex has a 
distorted five-coordinate geometry (Stephens \& Tucker, 1973) and retention of the bound chloride in nitromethane is confirmed by conductivity measurements. The similarity of the ligand-field transitions in solution UV-Vis spectra of the salt in nitromethane (Ondrejovic et al., 2002) and PBS support the retention of a distorted five-coordinate geometry in both solvents. The pre-edge spectra of $\left[\mathrm{CuCl}(\mathrm{bpy})_{2}\right]^{+}$have a weak feature near $8978 \mathrm{eV}$ (Fig. 4) assigned to the 'Laporte forbidden' $1 s-3 d$ transition of copper(II) (Tomson et al., 2015; DuBois et al., 2000; Kau et al., 1987). Photoreduction, with the generation of copper(I) complexes, is associated with the appearance of the more intense $1 s-4 p$ transition, which has been reported in the range $8983-8986 \mathrm{eV}$, depending on the coordination of the metal (Kau et al., 1987). The increase in intensity at $c a 8982 \mathrm{eV}$ with the unattenuated beam (Fig. 4 inset) can be attributed to the photoreduction of $\left[\mathrm{CuCl}(\mathrm{bpy})_{2}\right]^{+}$. It is noteworthy that the changes to the postedge oscillations (e.g. at $c a 9020 \mathrm{eV}$ ) due to photoreduction are more pronounced than that of the $8982 \mathrm{eV}$ feature (Fig. 4).

If the maximum safe flux is insufficient for the collection of extended XAS spectra with a good signal-to-noise ratio then the flow parameters can be further refined. In contrast to measurements from a static sample, the flowing solution will have a fixed, steady-state composition and will be less affected by spectral distortion resulting from time-dependent changes in sample composition induced by prolonged exposure to the beam (Best et al., 2016; Streltsov et al., 2018).

The surfaces of the WE may be contaminated by deposition of insoluble products from electrolysis or photolysis and this can lead to significant time-dependent changes in the spectra. For metalloproteins and polypeptides this may include oligomerization by coupling of radical species generated at the protein surface, or through protein adsorption on the electrode surface. It is common for the electrochemical response

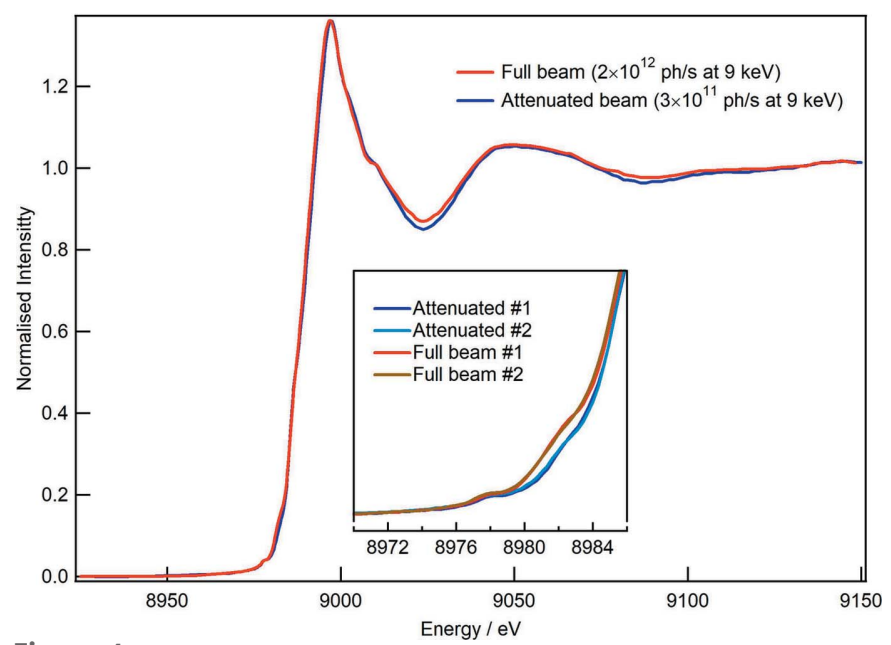

Figure 4

Power-dependence of the XANES of $\left[\mathrm{CuCl}(\mathrm{bpy})_{2}\right] \mathrm{Cl}$-hydrate $(2 \mathrm{~m} M$ in PBS). Pairs of normalized spectra are shown for measurements recorded with full beam and attenuated beam using $0.2 \mathrm{~mm} \mathrm{Al}$ foil. Further increases in beam attenuation did not result in significant spectral changes. The inset shows pairs of spectra recorded obtained with full and attenuated beams and highlights the reproducibility of the spectral changes. of the cell to change with increased exposure of the electrode to the protein solution. This will often be the result of adsorption of fragments of apo-protein and will not significantly impact the XAS spectra. The adsorption of metalloprotein will, however, have a significant adverse impact, particularly when there is adsorption at the point of exposure to the X-ray beam. Although instances of this effect will generally manifest as a continuous increase in the edge intensity with time, more subtle effects can only be checked at the end of data collection when the sample solution can be replaced by supporting electrolyte. In this case the loss of all signal from the element of interest provides confirmation that the measured spectra are not contaminated by surfaceadsorbed species. Where the voltametric or spectroscopic signatures suggest possible contamination of the WE it is important to compare the in situ measurements with spectra obtained from solution or frozen samples that do not include the RVC WE.

\subsection{Electrochemistry}

As noted previously, despite the large area of the WE, reasonable electrochemical response can be obtained from XAS-SEC cells if due attention is given to ion migration and the geometry of the CE and RE (Best et al., 2016). Precise control over the placement of the electrodes for the 3D printed cell allows further improvement in performance. Herein, we focus on the beam-dependent response of the XAS-SEC cell and the insight this can give on the chemical potential of the solution under investigation during continuous illumination by ionizing radiation. In a previous study it was reported that the magnitude and sign of the photoinduced current response from an electrode-adsorbed film of $\mathrm{CoO}_{x}$ in XAS-SEC experiments was sensitive to the applied potential (King et al., 2019). The sign of the photocurrent was interpreted in terms of oxidation or reduction of the $\mathrm{CoO}_{x}$ film as a consequence of photoionization.

In aqueous solution photoionization leads to strongly oxidizing and reducing species, namely aquated electrons, hydrogen and hydroxy radicals which are rapidly consumed in annihilation (e.g. formation of $\mathrm{H}_{2}$ or $\mathrm{H}_{2} \mathrm{O}_{2}$ ) and spontaneous redox reactions with solute species (Schwarz, 1955) which include, but are not limited to, the copper complex (Anbar \& Neta, 1967). In active modes of operation of the electrochemical cell, the WE will act as a redox participant and will provide a sink for both highly oxidizing and reducing species and maintain the Nernst equilibrium for the chemically reversible redox couples. The high rates of reaction of the initial products of photoionization relative to mass transport make the WE a less probable redox partner than highconcentration solute species. Enhanced protection against photodamage of the sample by in situ electrochemical approaches is mostly derived from the removal of the longerlived reactive secondary products of photoionization and reestablishment of the Nernst equilibrium of the species of interest. Electrochemically irreversible reactions involving such species may be chemically reversed by longer exposure 
of the solution to the WE away from the irradiation zone and, under these circumstances, the sample solution may be sampled multiple times without significant change in the spectra (Streltsov et al., 2018).

In open-circuit operation the potential of the WE relative to the RE corresponds to the open-circuit potential (OCP). This will relate to the relative concentrations and redox potentials of the solute species in contact with the WE and is a measure of the $E_{\mathrm{h}}$ of the solution. Since only ca $1 \%$ of the WE is subject to illumination by ionizing radiation, the OCP will not give a quantitative measure of the solution $E_{\mathrm{h}}$ in the illumination zone. However, the change in OCP in the absence and presence of illumination will be indicative of the change in $E_{\mathrm{h}}$ of the solution under investigation which, in turn, can be used to assess the susceptibility of the sample to oxidation or reduction during measurement.

The OCP of a $2 \mathrm{mM}$ solution of $\left[\mathrm{CuCl}(\mathrm{bpy})_{2}\right]^{+}$in $\mathrm{PBS}$ in the XAS-SEC cell with a fresh RVC WE was $c a 0.35 \mathrm{~V}$ versus $\mathrm{Ag} / \mathrm{AgCl}$. The OCP appears to be highly sensitive to exposure of the sample to the beam as illustrated by measurements obtained from the last spectrum of a series recorded with attenuated- and full-beam intensity (Fig. 5). The OCP value depends on the concentration and chemical activity of the oxidized and reduced products and, together with the conditioning of the electrode, this depends on the beam intensity and the flow properties of the solution. The negative shift of the OCP indicates more reducing conditions and is consistent with the reported proclivity of copper(II) complexes to photoreduction (Summers et al., 2019; Stellato et al., 2019). The response of the OCP to a change in beam intensity is reflected by the highly reproducible signature associated with the start of each scan. This coincides with a check of the alignment of the four-bounce monochromator axes during which the beam on the sample is interrupted in a highly

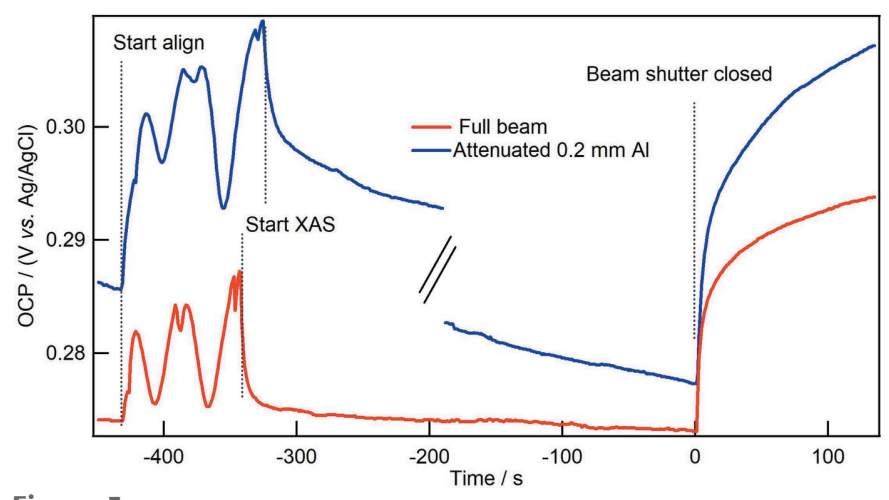

Figure 5

Variation of the open-circuit potential (OCP) with time during measurement of the XANES of $\left[\mathrm{CuCl}(\mathrm{bpy})_{2}\right]^{+}$(Fig. 4). The timescale for the full-beam trace was adjusted so that the shutter is closed at time $t=0 \mathrm{~s}$. Prior to the start of each scan the alignment of the four-bounce monochromator axes is checked, and this gives a reproducible set of variations in the beam intensity. A wider scan range was applied for the attenuated-beam scan. The start and end of the full- and attenuated-beam measurements were aligned by extracting a block of measurements during the longer attenuated-beam scan. The full OCP data for the attenuated-beam measurements and a more detailed description of the monochromator alignment routine are given in Section S4 of the supporting information. reproducible manner (Fig. 5). The continued decrease in OCP on resumption of continuous illumination reflects an increased concentration of reduced species in contact with the electrode, but outside the illumination zone, and is a result of the net flow of the solution through the WE. As expected, these effects are more pronounced for the full beam. Closing the incident beam shutter is accompanied by an increase in the OCP which can be modelled to a double exponential decay (Section S4 of the supporting information). This is consistent with a combination of more short-lived products (half-life $<5 \mathrm{~s}$ ), which will be restricted to the solution under illumination, and longer-lived products (half-life $>40 \mathrm{~s}$ ), which will be more dispersed within the WE. For measurements with a higher photon flux the decay results in a more negative value of the OCP, suggesting a greater change in the composition of the solution as a result of irreversible chemical reactions. Although the available measurements do not allow a more quantitative interpretation of the OCP measurements, they do highlight the potential that such measurements can provide for understanding the sensitivity of solution samples to photoionization, and assess the impact of additives such as cryoprotectants or redox mediators on the redox stability of the solution.

\section{Conclusions}

The developments in the cell design and implementation of XAS-SEC techniques have led to significant improvements in the electrochemical performance, reliability, minimization of sample volume and quality of the resulting XAS spectra. Key to the successful implementation of the approach is the control of solution flow through the WE/sample cell. Optimization of the pulse stroke and net flow rate parameters and beam intensity allow the collection of high-quality spectra from comparatively modest solution volumes. The electrochemical cell provides control over both the relative concentrations of the redox forms of the sample and a sink for strongly oxidized and reduced species formed following photoionization and, in this way, can 'heal' the sample following exposure to the X-ray beam. Following optimization of the flow parameters it is possible to flow a small volume (e.g. $100 \mu \mathrm{l})$ of the solution repeatedly ( $>10$ times, over a period of several hours) through the WE without significant change to the spectra and this allows measurement from solution volumes as low as $600 \mu \mathrm{l}$.

While XAS-SEC measurements are undoubtedly more demanding than those conducted from frozen solutions, there are significant payoffs in terms of identification, minimization and control of photoreduction as well as control over the redox state. Moreover, interference from Bragg peaks - a persistent blight of cryogenic measurements - is absent for solution samples. The measurement is dynamic: the spectroscopic response to changes of potential, solution flow and photon flux feed into experiment design and better connect to the chemistry of the system. Finally, there is, of course, a significant advantage to be gained by conducting measurements from systems with acid or base functionality, such as 
metalloproteins, with control over both the $\mathrm{pH}$ and temperature.

The advances in 3D printing and more general accessibility to commercial printing and computer-controlled machining and cutting services have brought low-volume purposedesigned and built apparatus into easy reach for researchers not blessed by unlimited budgets or subsidised workshops. The current publication provides details needed for construction and operation of the apparatus for XAS-SEC measurements. The opportunity to refine and easily test design modifications allows the optimization of experiments which it is hoped will contribute to the study of more challenging systems.

\section{Acknowledgements}

This work was carried out with the support of the Diamond Light Source, instrument I20-Scanning (proposal Nos. SP20589 and SP23523). SPB thanks Michael Zammit and Alexandre Tolotchkov of the Science Faculty workshop for expert technical assistance with the mechanical and electronic equipment, and Oktay Balkis and the mechanical workshop of the University of Melbourne for assistance with 3D printing and laser cutting. The opensource program OpenSCAD provided a path to development of the $3 \mathrm{D}$ printed cells and we are grateful for the availability of that resource. During the experiment we benefitted from the technical support provided by the Spectroscopy group technicians and, in particular, by Darren Neville.

\section{Funding information}

CTC gratefully acknowledges the University of Melbourne for a grant which includes travel support. SPB and VAS gratefully acknowledge travel funding provided by the International Synchrotron Access Program (ISAP) managed by the Australian Synchrotron (award No. 16027), part of ANSTO and funded by the Australian Government.

\section{References}

Anbar, M. \& Neta, P. (1967). Int. J. Appl. Radiat. Isot. 18, 493-523. Best, S. P., Levina, A., Glover, C., Johannessen, B., Kappen, P. \& Lay, P. A. (2016). J. Synchrotron Rad. 23, 743-750.

Bourke, J. D., Islam, M. T., Best, S. P., Tran, C. Q., Wang, F. \& Chantler, C. T. (2016). J. Phys. Chem. Lett. 7, 2792-2796.

Bunker, G. (2010). Introduction to XAFS: a Practical Guide to X-ray Absorption Fine Structure Spectroscopy. Cambridge University Press.

Chatterjee, R., Weninger, C., Loukianov, A., Gul, S., Fuller, F. D., Cheah, M. H., Fransson, T., Pham, C. C., Nelson, S., Song, S., Britz, A., Messinger, J., Bergmann, U., Alonso-Mori, R., Yachandra, V. K., Kern, J. \& Yano, J. (2019). J. Synchrotron Rad. 26, 1716-1724. Cotelesage, J. J. H., Pushie, M. J., Grochulski, P., Pickering, I. J. \& George, G. N. (2012). J. Inorg. Biochem. 115, 127-137.

Dennis, G., Helsby, W., Omar, D., Horswell, I., Tartoni, N., Hayama, S., Mikulska, I. \& Diaz-Moreno, S. (2019). AIP Conf. Proc. 2054, 060065 .
Diaz-Moreno, S., Amboage, M., Basham, M., Boada, R., Bricknell, N. E., Cibin, G., Cobb, T. M., Filik, J., Freeman, A., Geraki, K., Gianolio, D., Hayama, S., Ignatyev, K., Keenan, L., Mikulska, I., Mosselmans, J. F. W., Mudd, J. J. \& Parry, S. A. (2018). J. Synchrotron Rad. 25, 998-1009.

DuBois, J. L., Mukherjee, P., Stack, T. D. P., Hedman, B., Solomon, E. I. \& Hodgson, K. O. (2000). J. Am. Chem. Soc. 122, 5775-5787.

Frenkel, A. I. \& van Bokhoven, J. A. (2014). J. Synchrotron Rad. 21, 1084-1089.

George, G. N., Pickering, I. J., Pushie, M. J., Nienaber, K., Hackett, M. J., Ascone, I., Hedman, B., Hodgson, K. O., Aitken, J. B., Levina, A., Glover, C. \& Lay, P. A. (2012). J. Synchrotron Rad. 19, 875-886. Grunwaldt, J.-D. \& Clausen, B. S. (2002). Top. Catal. 18, 37-43.

Hayama, S., Duller, G., Sutter, J. P., Amboage, M., Boada, R., Freeman, A., Keenan, L., Nutter, B., Cahill, L., Leicester, P., Kemp, B., Rubies, N. \& Diaz-Moreno, S. (2018). J. Synchrotron Rad. 25, 1556-1564.

Kau, L. S., Spira-Solomon, D. J., Penner-Hahn, J. E., Hodgson, K. O. \& Solomon, E. I. (1987). J. Am. Chem. Soc. 109, 6433-6442.

King, H. J., Fournier, M., Bonke, S. A., Seeman, E., Chatti, M., Jumabekov, A. N., Johannessen, B., Kappen, P., Simonov, A. N. \& Hocking, R. K. (2019). J. Phys. Chem. C, 123, 28533-28549.

Kubin, M., Guo, M., Ekimova, M., Baker, M. L., Kroll, T., Källman, E., Kern, J., Yachandra, V. K., Yano, J., Nibbering, E. T. J., Lundberg, M. \& Wernet, P. (2018). Inorg. Chem. 57, 5449-5462.

Ondrejovic, G., Kotocova, A. \& Valigura, D. (2002). Chem. Pap. 56, 168-173.

OpenSCAD (2020). OpenSCAD, http://www.openscad.org/index. html.

Parsons, J. G., Aldrich, M. V. \& Gardea-Torresdey, J. L. (2002). Appl. Spectrosc. Rev. 37, 187-222.

Schalken, M. J. \& Chantler, C. T. (2018). J. Synchrotron Rad. 25, 920 934.

Schultz, F. A., Feldman, B. J., Gheller, S. F., Newton, W. E., Hedman, B., Frank, P. \& Hodgson, K. O. (1993). Redox Mechanisms and Interfacial Properties of Molecules of Biological Importance, Vol. 93-11, edited by F. A. Schultz and I. Taniguchi, pp. 108-117. Pennington: The Electrochemical Society.

Schwarz, H. A. (1955). J. Am. Chem. Soc. 77, 4960-4964.

Shima, S., Pilak, O., Vogt, S., Schick, M., Stagni, M. S., Meyer-Klaucke, W., Warkentin, E., Thauer, R. K. \& Ermler, U. (2008). Science, 321, 572-575.

Stellato, F., Chiaraluce, R., Consalvi, V., De Santis, E., La Penna, G., Proux, O., Rossi, G. \& Morante, S. (2019). Metallomics, 11, 14011410.

Stephens, F. S. \& Tucker, P. A. (1973). J. Chem. Soc. Dalton Trans. pp. 2293-2297.

Streltsov, V. A., Ekanayake, R. S. K., Drew, S. C., Chantler, C. T. \& Best, S. P. (2018). Inorg. Chem. 57, 11422-11435.

Streltsov, V. A., Titmuss, S. J., Epa, V. C., Barnham, K. J., Masters, C. L. \& Varghese, J. N. (2008). Biophys. J. 95, 3447-3456.

Summers, K. L., Schilling, K. M., Roseman, G., Markham, K. A., Dolgova, N. V., Kroll, T., Sokaras, D., Millhauser, G. L., Pickering, I. J. \& George, G. N. (2019). Inorg. Chem. 58, 6294-6311.

Tamura, H., Salomone-Stagni, M., Fujishiro, T., Warkentin, E., Meyer-Klaucke, W., Ermler, U. \& Shima, S. (2013). Angew. Chem. Int. Ed. 52, 9656-9659.

Tomson, N. C., Williams, K. D., Dai, X., Sproules, S., DeBeer, S., Warren, T. H. \& Wieghardt, K. (2015). Chem. Sci. 6, 2474-2487.

Trevorah, R. M., Chantler, C. T. \& Schalken, M. J. (2020). J. Phys. Chem. A, 124, 1634-1647.

Zhang, H. H., Hedman, B. \& Hodgson, K. O. (1999). Inorganic Electronic Structure and Spectroscopy, pp. 513-554. New York: John Wiley.

Zhang, L. (2019). Methods Mol. Biol. 1876, 179-195. 